\title{
TU/e EN⿴HONE

\section{Scaling of current distributions in variable-range hopping transport on two- and three-dimensional lattices}

\section{Citation for published version (APA):}

Pasveer, W. F., Bobbert, P. A., Huinink, H. P., \& Michels, M. A. J. (2005). Scaling of current distributions in variable-range hopping transport on two- and three-dimensional lattices. Physical Review B, 72(17), 174204-1/6. [174204]. https://doi.org/10.1103/PhysRevB.72.174204

DOI:

10.1103/PhysRevB.72.174204

Document status and date:

Published: 01/01/2005

\section{Document Version:}

Publisher's PDF, also known as Version of Record (includes final page, issue and volume numbers)

\section{Please check the document version of this publication:}

- A submitted manuscript is the version of the article upon submission and before peer-review. There can be important differences between the submitted version and the official published version of record. People interested in the research are advised to contact the author for the final version of the publication, or visit the $\mathrm{DOI}$ to the publisher's website.

- The final author version and the galley proof are versions of the publication after peer review.

- The final published version features the final layout of the paper including the volume, issue and page numbers.

Link to publication

\section{General rights}

Copyright and moral rights for the publications made accessible in the public portal are retained by the authors and/or other copyright owners and it is a condition of accessing publications that users recognise and abide by the legal requirements associated with these rights.

- Users may download and print one copy of any publication from the public portal for the purpose of private study or research.

- You may not further distribute the material or use it for any profit-making activity or commercial gain

- You may freely distribute the URL identifying the publication in the public portal.

If the publication is distributed under the terms of Article 25fa of the Dutch Copyright Act, indicated by the "Taverne" license above, please follow below link for the End User Agreement:

www.tue.nl/taverne

Take down policy

If you believe that this document breaches copyright please contact us at:

openaccess@tue.nl

providing details and we will investigate your claim. 


\title{
Scaling of current distributions in variable-range hopping transport on two- and three-dimensional lattices
}

\author{
W. F. Pasveer, P. A. Bobbert, H. P. Huinink, and M. A. J. Michels \\ Group Polymer Physics, Eindhoven Polymer Laboratories and Dutch Polymer Institute, Technische Universiteit Eindhoven, \\ P.O. Box 513, NL-5600 MB Eindhoven, The Netherlands
}

(Received 14 July 2005; revised manuscript received 22 September 2005; published 16 November 2005)

\begin{abstract}
From extensive computer simulations of variable-range hopping (VRH) transport of charges on regular twoand three-dimensional lattices with random site energies we calculate the average contribution to the total current of hops over a certain distance and with a certain hop energy. We find that the resulting current distribution is a universal function of scaled distance and energy variables. We discuss this scaling in the light of the original arguments of Mott and percolation arguments to explain the temperature dependence of the VRH conductivity.
\end{abstract}

DOI: 10.1103/PhysRevB.72.174204

PACS number(s): 72.20.Ee, 89.75.Da, 78.55.Qr, 82.20.Wt

\section{INTRODUCTION}

Many experimental and theoretical studies have by now established the fact that the low-temperature conductivity $\sigma$ in many disordered semiconductors deviates from an Arrhenius behavior and obeys a temperature $(T)$ dependence of the form

$$
\sigma=\sigma_{0} \exp \left[-\left(T_{0} / T\right)^{\gamma}\right],
$$

with $\gamma<1$ and $\sigma_{0}$ a prefactor that depends weakly (algebraically) on temperature and other system parameters. In the early days, this non-Arrhenius behavior was studied in $\mathrm{Si}$ and Ge-based systems, III-V compounds, and glasses. ${ }^{1}$ More recently, this behavior has been found in completely different systems, sich as high- $T_{c}$ cuprate oxides, ${ }^{2}$ conjugated polymers, ${ }^{3,4}$ and DNA. ${ }^{5}$ Experimentally, it has been recognized that $\gamma$ can take on the discrete values $\gamma \approx 0.25,0.33$, and 0.50 .

Shortly after the observations of this behavior, Mott ${ }^{6}$ presented an explanation based on so-called variable-range hopping (VRH) between localized states. The central idea of this explanation is that in disordered systems charge carriers hop from one localized site to another and that at low temperatures the hopping from a particular site takes place over variable distances to sites with a more favorable energy than that of the sites close by. In particular, Mott showed that ${ }^{6}$

$$
k_{\mathrm{B}} T_{0}=c_{d} \alpha^{d} / g, \quad \gamma=1 /(d+1),
$$

with $c_{d}$ a constant of order unity, $\alpha \equiv 2 / a,{ }^{7} a$ the localization radius of the wave functions, $g$ the (constant) density of states around the Fermi level, and $d$ the spatial dimension. In his explanation Mott considered the transition rate between two localized sites derived by Miller and Abrahams ${ }^{8}$

$$
W_{i j}= \begin{cases}\nu_{0} \exp \left[-\alpha R_{i j}-\beta\left(E_{j}-E_{i}\right)\right], & E_{j} \geqslant E_{i}, \\ \nu_{0} \exp \left[-\alpha R_{i j}\right], & E_{j}<E_{i},\end{cases}
$$

where $\beta \equiv 1 / k T, \nu_{0}$ is an intrinsic rate, and $R_{i j} \equiv\left|\mathbf{R}_{j}-\mathbf{R}_{i}\right|$ is the distance between sites $i$ and $j$, with energies $E_{i}$ and $E_{j}$, respectively. This expression accounts both for tunneling through a region in which the tail of the wave function of the localized states can be approximated by an exponential, ${ }^{9}$ and for jumps upward in energy via phonon-assisted thermal activation. Equation (3) can be derived under the assumption of coupling to a system of acoustical phonons, and the conditions that the thermal energy $k_{\mathrm{B}} T$ is small compared to the energy differences $\left|E_{i}-E_{j}\right|$ and that these energy differences are smaller than, or of the order of, the Debye energy. ${ }^{8}$ The central argument in the explanation by Mott is that if a charge carrier hops over a distance less than $R$, the average spacing of energy levels within that distance is $\Delta E \sim g / R^{d}$, which is therefore the typical activation energy. The typical hop that determines the conductivity is obtained by maximizing $\exp [-\alpha R-\beta \Delta E]$ with respect to $R$ or $\Delta E$. Equations (1) and (2) are then readily obtained.

Early on already ${ }^{10-12}$ the argument by Mott was criticized, and in particular the focus on one typical hop, and attempts were made to base Eqs. (1) and (2) on percolation theory. In the framework of percolation theory a mapping is made onto a random-resistor network and a conductance is attributed to each pair of hopping sites, which, for low temperatures, becomes $^{10}$

$$
G_{i j}=\frac{e^{2}}{k T} \nu_{0} \exp \left[-\alpha R_{i j}-\beta E_{i j}\right]
$$

with $e$ the electronic charge and

$$
E_{i j} \equiv\left(\left|E_{i}\right|+\left|E_{j}\right|+\left|E_{j}-E_{i}\right|\right) / 2 .
$$

Bonds are now artificially introduced in decreasing order of conductance until a percolating network is established at a critical conductance $G_{c}$. This critical conductance is supposed to determine the overall conductance of the system at low temperatures. The idea is that the addition of bonds with lower conductances than $G_{c}$ does not change the conductivity of the system anymore since these bonds are short circuited by the already present bonds with higher conductances. The prediction of percolation theory for the exponential factor in Eq. (1) is therefore supposed to be exact in the limit of vanishing temperature. ${ }^{13}$

However, precisely at the percolation point there will be a dramatic difference between the real network, which has a nonzero and size-independent conductivity, and the fractal 
network with the short-circuited bonds ignored, which has a size-dependent and vanishing conductivity. To account for this difference, percolation theory has to be extended, considering bonds beyond the percolation point with conductances lower than $G_{c}$ in, e.g., the "nodes-links-blobs" model. ${ }^{12,14-16}$ This extension of percolation theory leads to an estimate for the prefactor $\sigma_{0}$ of the conductivity in Eq. (1). ${ }^{12}$

Although the essential correctness of the VRH functional form Eq. (1) has been firmly established, both the theoretical basis and the interpretation of the experimentally accessible and morphology-related parameters such as $T_{0}$ have remained topics of discussion. This also applies to extensions of the theory, e.g., to include Coulomb effects and nonconstant densities of states, and contributions to the theory are still appearing. ${ }^{3,4,17-21}$ Also, effective-medium theory was applied to the VRH problem, ${ }^{22-24}$ leading to results comparable to percolation theory. For overviews of the theory and applications of VRH, we refer the reader to textbooks, e.g., Refs. 12,25 , and 26.

Explicit calculations of conductances of random-resistor networks by solving Kirchhoff's equations were performed in the $1970 \mathrm{~s},{ }^{27-29}$ the results of which were reported to be in reasonable agreement with analytical formulas derived from percolation theory. ${ }^{29}$ Surprisingly, the numerical approach has not been much pursued, despite the formidable increase of computer power. In the present work we choose to solve the VRH problem numerically exactly, without mapping to a resistor network. Moreover, the system sizes and number of disorder configurations we can handle are much larger than in the numerical work of the 1970s. Apart from the determination of the overall conductivity of two- and threedimensional VRH systems, this allows us to make an accurate study of the currents through bonds characterized by certain distances $R_{i j}=R$ and energies $E_{i j}=E$.

\section{METHOD}

Neglecting electron-electron correlations, except for the requirement that only one electron can occupy a single site, and assuming thermal equilibrium, the average occupation probabilities $n_{i}$ of the hopping sites $i$ follow from Boltzmann's equation

$$
\sum_{j \neq i}\left[W_{i j} n_{i}\left(1-n_{j}\right)-W_{j i} n_{j}\left(1-n_{i}\right)\right]=0 .
$$

We solve this set of equations for the occupation probabilities $n_{i}$ with transition rates Eq. (3) on square lattices in two and three dimensions with lattice constant $R_{0}$, in the presence of an electric field $\mathbf{E}$. The lattice site energies are drawn randomly from a homogeneous distribution on the interval $\left[-E_{0} / 2, E_{0} / 2\right]$, leading to a density of states $g=\left(R_{0}^{d} E_{0}\right)^{-1}$. We assume that we are dealing with systems in which effects due to the Coulomb gap, leading to a vanishing density of states around the Fermi energy, ${ }^{12}$ are negligible. The energy differences in Eq. (3) are supposed to contain a contribution $-e \mathbf{E} \cdot\left(\mathbf{R}_{j}-\mathbf{R}_{i}\right)$ due to the electric field $\mathbf{E}$. In the limit of small electric fields we can linearize and transform Eq. (6) into a set of linear equations for the variables

$$
p_{i} \equiv\left(n_{i}-n_{i}^{0}\right) /\left[n_{i}^{0}\left(1-n_{i}^{0}\right)\right],
$$

where $n_{i}^{0}=\left[1+\exp \left(\beta E_{i}\right)\right]^{-1}$ is the equilibrium Fermi-Dirac distribution. We choose the Fermi energy $E_{\mathrm{F}}=0$. Due to the spatial exponential decay in the transition rates Eq. (3) very accurate results can be obtained by taking only a limited number of hopping sites $j$ around $i$ into account. In addition, this allows us to use periodic boundary conditions not only in the direction(s) perpendicular to the electric field, but along the electric field as well. Using standard sparse matrix routines, we are able to treat very large systems at low temperatures by our algorithm.

From the occupation probabilities $n_{i}$ in the presence of an electric field, the current $I$ through the system, and from that the conductivity $\sigma$, is calculated by evaluating the net current between each pair of hopping sites and summing over the lattice. In addition, we determine a current distribution $I(R, E)$ by calculating the average current flowing into the direction of the electric field between pairs of sites with mutual distance $R_{i j}=R$ (implying an angular average) and energy $E_{i j}=E$ as defined by Eq. (5). On our regular lattices only discrete values of $R$ are possible. The energy interval $\left[0, E_{0}\right]$ is divided into a convenient number of "bins" (typically of the order of 100), such that a reliable average is obtained together with a high enough resolution. Averages are determined over a sufficient number of disorder realizations and the system sizes are taken large enough to avoid finite size effects. The typical system sizes used in our calculations were $100 \times 100$ for $d=2$ and $50 \times 50 \times 50$ for $d=3$. The maximum hop distance taken into account was $12 R_{0}$ for $d$ $=2$ and $5 R_{0}$ for $d=3$, hence much smaller than the system size, but large enough to allow for far enough hops in the parameter range studied (see the next section).

\section{RESULTS}

In Fig. 1 we plot the conductivity $\sigma$ for dimensions $d=2$ and $d=3$ for $\alpha=4 / R_{0}$ logarithmically versus the dimensionless variable

$$
l \equiv\left[\alpha^{d} \beta / g\right]^{1 /(1+d)} .
$$

We clearly observe the validity of Eq. (1) at low temperatures from the fact that the data points tend to a straight line. We also plot in Fig. 1 lines with a slope following from the calculations of Skal and Shlovskii, ${ }^{30}$ who solved the percolation problem numerically, leading to $c_{2}=1.51 \pm 0.03$ and $c_{3}=1.28 \pm 0.02$. Assuming the percolation treatment of Skal and Shklovskii ${ }^{30}$ to yield the correct exponential factor in Eq. (1) in the limit $T \rightarrow 0$, the difference in slopes between the numerical and percolation results should be attributed to the algebraic temperature dependence of the preexponential factor $\sigma_{0}$ in Eq. (1). We did not attempt to come to a quantification of this preexponential factor (see, e.g., Ref. 12 for a discussion), since this is not the main subject of this work. On the other hand, somewhat different values of $c_{2}$ and $c_{3}$ can also be found in the literature (compiled in, e.g., Ref. 25, which makes the comparison complicated.

In Fig. 2 we plot the current distributions $I(R, E)$. We clearly see that most of the current is not carried by the 


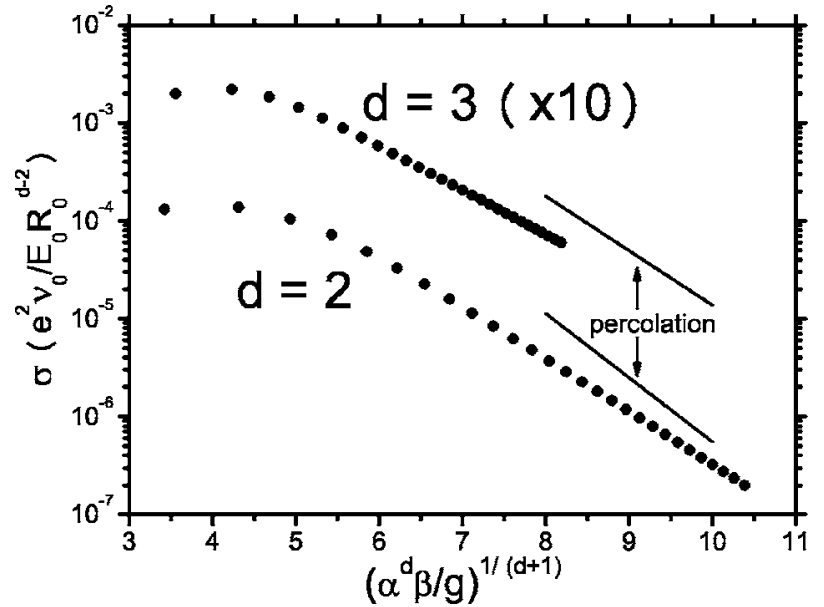

FIG. 1. VRH conductivity for $\alpha=4 / R_{0}$ in dimensions $d=2$ $(100 \times 100$ array $)$ and $d=3(50 \times 50 \times 50$ array $)$. The maximum hopping distance is $12 R_{0}\left(5 R_{0}\right)$ and averages have been taken over 40 (8) disorder configurations for $d=2(d=3)$. The error bars are not visible on this scale. The straight lines represent the lowtemperature slopes as predicted by percolation theory.

high-conductance bonds near $R=E=0$, but by bonds near a peak $R=R_{\max }, E=E_{\max }$. A striking universal behavior of the distributions $I(R, E)$ is observed when $\alpha$ and $\beta$ are varied. In the second and third pair of panels of Fig. 2, as counted from above, $\beta$ has been changed from $\beta=67.5 / E_{0}$ in the upper two panels to $\beta=37.5 / E_{0}$. In the fourth and fifth pair of panels, $\alpha$ has been changed from $\alpha=4 / R_{0}$ to $\alpha=3 / R_{0}$. When the functions $I(R, E)$ for different $\beta$ and $\alpha$ are plotted versus the variables

$$
\begin{aligned}
& \rho \equiv \alpha R / l, \\
& \epsilon \equiv \beta E / l,
\end{aligned}
$$

these functions appear to collapse onto a master function $f(\rho, \epsilon)$, apart from a prefactor proportional to the total current [the integral of $I(R, E)$ over $R$ and $E$ ]. In all the panels of Fig. 2 the top and right axes indicate the unscaled variables $R / R_{0}, E / E_{0}$, and the bottom and left axes the scaled variables $\rho, \epsilon$, respectively. In the third and fifth pair of panels, we have scaled the axes to demonstrate the universal behavior. We conjecture that the small observable differences should be attributed to the fact that at the temperatures for which the calculations have been done the discrete nature of the lattice still plays a role and that these differences will vanish in the limit $T \rightarrow 0$, when

$$
R_{\max } \gg R_{0}, \quad E_{\max } \gg k_{\mathrm{B}} T, \quad E_{\max } \ll E_{0} .
$$

If these conditions are obeyed, also the type of lattice becomes irrelevant and the site positions may become random, as in realistic situations. We note that the first and second condition in Eq. (11) are not really satisfied in our calculations ( $>$ applies rather than $\gg$ ) and that it is in some sense surprising that the universal behavior is nevertheless so clearly observed. We could not reach lower temperatures than $k_{\mathrm{B}} T \approx 0.013 E_{0}$, due to numerical instabilities. We should say that the master functions for $d=2$ and $d=3$ in Fig. 2 look similar, but that we have no reason to suspect that they are the same. We note that the usefulness of the dimensionless variables Eqs. (9) and (10) as the natural variables to express the problem was realized before, e.g., in the effectivemedium treatment of Ref. 23. From Fig. 2 it is clear that $R_{\max }$ is small compared to the maximum hopping distance allowed in our calculations $\left(12 R_{0}\right.$ for $d=2$ and $5 R_{0}$ for $\left.d=3\right)$ and that $E_{\max }$ is small compared to the cut-off energy $E_{0}$ of our density of states.

By the drawn straight lines in Fig. 2 we indicate the value $\alpha R+\beta E$ for which the conductance following from Eq. (4) is equal to the critical percolating conductance $G_{c}$, as calculated by Skal and Shklovskii. ${ }^{30}$ According to percolation theory, the bonds with $G<G_{c}$ (to the right/above the straight lines) are short circuited by the bonds with $G>G_{c}$ and hence the relative contribution of those bonds to the total current should vanish in the limit $T \rightarrow 0$. Such behavior appears to be compatible with the plots for $d=2$. For $d=3$ there still appears to be a substantial contribution to the current of bonds for which $G<G_{c}$.

\section{CONCLUSIONS AND DISCUSSION}

The main conclusion from this work is that in VRH the current distribution $I(R, E)$ obeys the following lowtemperature scaling relation:

$$
I(R, E) \sim g I f(\rho \equiv \alpha R / l, \epsilon \equiv \beta E / l),
$$

where $I$ is the total current through the system and $f(\rho, \epsilon)$ is a universal function, i.e., a function that does not depend on the parameters of the system.

The expression Eq. (12) suggests the interpretation of $l$, defined by Eq. (8), as a dimensionless scale parameter, both in space and energy. Indeed, it can be demonstrated that at low temperatures the parameter $l$ is the only relevant parameter of the system. It is easy to see that exactly the same mathematical problem is solved if the density of states $g$ is varied, $g \rightarrow g^{\prime}=s g$, and at the same time the temperature, $\beta$ $\rightarrow \beta^{\prime}=s \beta$, leaving $l$ unchanged. Hence, the problem is exactly invariant under this transformation. It can also be demonstrated that at low temperatures the problem is invariant under the following spatial scale transformation. Suppose we apply a transformation to the system for $d=2(d=3)$ by grouping the sites in squares (cubes) with side $2 R_{0}$. The site within each square (cube) that is best connected to sites in other squares (cubes) is the one closest to the Fermi energy and we select this site in each square (cube). We construct a system with lattice constant $2 R_{0}$ consisting of these selected sites, see Fig. 3 for the case $d=2$. If the conditions Eq. (11) are satisfied before and after the transformation, we can expect that the large-scale current distribution of the original system is comparable to that of the new system. It is easily seen that the density of states around the Fermi energy in the new system is equal to $g^{\prime}=2^{d} g$. This system can also be viewed as a system with the original lattice constant $R_{0}$, but with a scaled $\alpha^{\prime}=2 \alpha$. Hence, we have established a transformation $\alpha \rightarrow \alpha^{\prime}=2 \alpha, g \rightarrow g^{\prime}=2^{d} g$ that leaves the problem invariant at low temperatures.

For $d=2$ we explicitly checked this scale transformation numerically. In Fig. 4 (left panel) we plot the electrochemical 

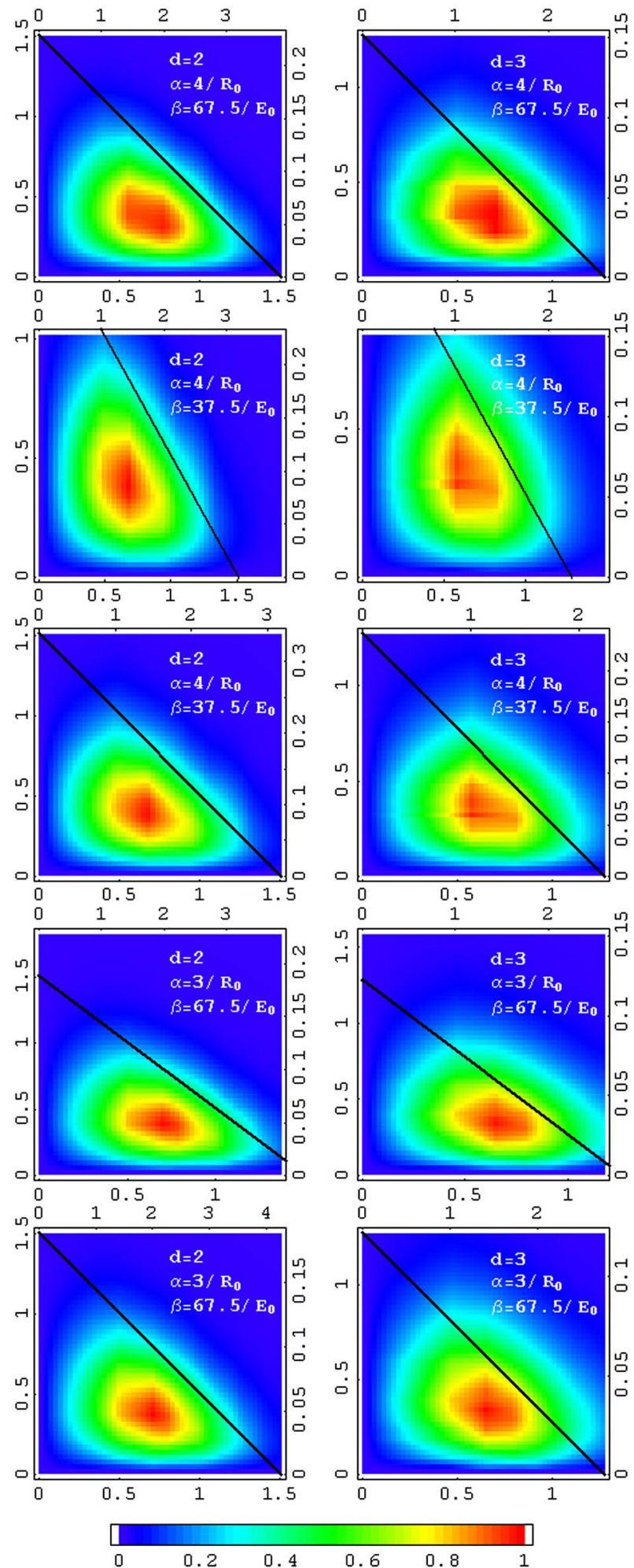

FIG. 2. (Color online) Current distributions $I(R, E)$ in $d=2$ (left panels) and $d=3$ (right panels). Bottom axes: $\rho$, left axes: $\epsilon$, top axes: $R / R_{0}$, right axes: $E / E_{0}$. Upper two panels: $\alpha=4 / R_{0}, \beta$ $=67.5 / E_{0}$, and average over 800 configurations. Other panels: changed $\beta$ or $\alpha$, with unscaled and scaled axes and average over 200 configurations. The displayed results are interpolations on a $R-E$ grid. Drawn lines: $R-E$ values of the critical conductance in percolation theory. The currents have been normalized by the maximum current and the bar gives the color code.

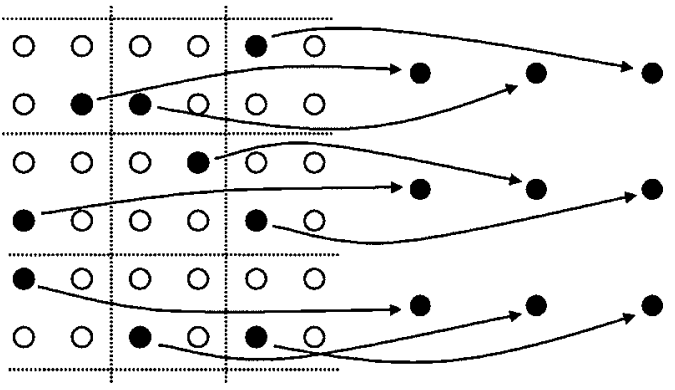

FIG. 3. Schematic representation of the transformation $\alpha$ $\rightarrow 2 \alpha, g \rightarrow 2^{d} g$ for $d=2$. The filled circles indicate the sites in the squares with energy closest to the Fermi energy.

potential in a $200 \times 200$ lattice for $\alpha=2 / R_{0}$ and $\beta=67.5 / E_{0}$, for a specific disorder configuration. In the low-temperature limit the electrochemical potential is related to the variables $p_{i}$ of Eq. (7) by

$$
\mu_{i}=k_{\mathrm{B}} T p_{i}-e \mathbf{E} \cdot \mathbf{R}_{i} .
$$

We also plot in Fig. 4 (right panel) the electrochemical potential in the $100 \times 100$ lattice for $\alpha=4 / R_{0}$ and $\beta=67.5 / E_{0}$ after the transformation, in which we have exactly followed the above prescription of site selection. We clearly see that the large-scale features in both potential distributions are equal and the same will hold for the current distributions. We conjecture that this invariance of the transformation becomes even better at lower temperatures.

It is evident that the scale transformation described above can be performed with different factors than 2, i.e., $\alpha \rightarrow \alpha^{\prime}$ $=s \alpha$ and $g \rightarrow g^{\prime}=s^{d} g$, and should lead to invariant current distributions if the conditions Eq. (11) are satisfied before and after the transformation. A combination of the two classes of transformations for which we have demonstrated invariance leads to all possible invariant transformations of $\alpha, \beta$, and $g$, for which $l$ is unchanged.

On the other hand, the scaling of $I(R, E)$ under a change of system parameters such that $l$ is changed is a nontrivial result and has not been reported before, as far as we know. What we can say is that this scaling is compatible with the
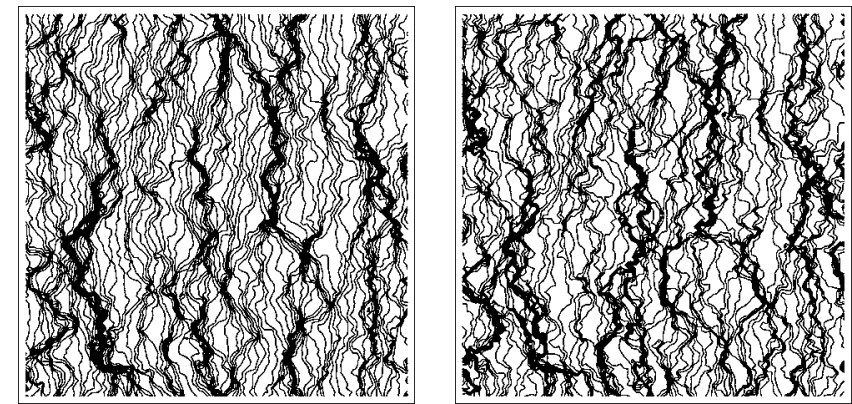

FIG. 4. Left: electrochemical potential in a $200 \times 200$ lattice with $\alpha=2 / R_{0}$ and $\beta=67.5 / E_{0}$, for a particular disorder configuration. Right: the same, but in the $100 \times 100$ lattice with $\alpha=4 / R_{0}$ after the transformation described in the main text. About 100 equipotential lines are drawn for a horizontal current. The conductivities are $6.32 \times 10^{-5}$ and $4.58 \times 10^{-5} e^{2} \nu_{0} / E_{0}$, respectively. 
original treatment of the VRH problem by Mott, as well as with the treatments based on percolation and effectivemedium theories, but that it is not necessarily a consequence of these treatments.

An interesting aspect of the scaling is that at decreasing temperatures the range over which the conductances Eq. (4) of the bonds in the regions displayed in the scaled plots of Fig. 2 vary becomes larger, whereas the range over which the scaled average currents through these bonds vary remains the same. This can only be understood if the electrochemical potential in the network organizes itself in such a way that at decreasing temperatures the ratio of the potential drops across bonds with low conductances and across bonds with high conductances becomes larger. Figure 4 gives a clue about how this could happen. The spatial structure of the electrochemical potential consists of terraces of almost constant potential, separated by cliffs at which the potential has sudden jumps. We observed that at decreasing temperatures the typical spacing between the cliffs and at the same time the typical potential jump across the cliffs become larger. We conjecture that bonds of low conductance will be clustered around the cliffs and bonds of high conductance around the terraces in such a way that this aspect of the scaling holds true.

The competition between the spatial and energy parts of the exponent in Eqs. (3) and (4), which is the essence of the original argument by Mott, ${ }^{6}$ is nicely demonstrated in Fig. 2. Most of the current goes through the bonds around the peak position in the $R-E$ plane. However, this does not mean that the overall conductivity is governed by these bonds. The essence of the percolation argument is that at low temperatures the overall conductivity is governed by the critical bonds with conductance $G_{c}$ (the straight lines in Fig. 2) at the percolation threshold. ${ }^{10-12}$ Bonds with conductance $G<G_{c}$ are short circuited by bonds with conductance $G>G_{c}$. For $d=2$ this appears to be compatible with our results. For $d$ $=3$ we find that there is still a considerable amount of current carried by bonds with conductances $G<G_{c}$. It is not clear to us what the reason is for this discrepancy. Assuming percolation theory to be exact in the limit $T \rightarrow 0$, a possibility is that, despite the apparent convergence obtained in Fig. 2, we still did not reach low enough temperatures in our calculations for $d=3$. Evidently, the calculations for $d=3$ are much more demanding than those for $d=2$ and the values for $l$ we can reach for $d=3$ are not as high as for $d=2$ (see Fig. 1). Another possibility is that the percolation factor $c_{3}$ $=1.28 \pm 0.02$ as calculated by Monte Carlo simulations ${ }^{30}$ has been underestimated.

The appearance of a peak at nonzero energy in Fig. 2 should not be confused with the concept of "transport energy." "31 This energy is supposed to maximize the hopping rate as a final energy in the hop between two localized states and to be independent of the initial energy. However, it was demonstrated by Baranovskii et al. that this concept is only useful for hopping in band tails with densities of states that decay fast enough as a function of energy. ${ }^{32}$ Hence, the concept is inapplicable to the situation of a constant density of states, as considered here. Furthermore, our definition Eq. (5) of the "hopping energy" depends on the final and initial energy.

The observation that the current distribution in VRH is determined by a universal master function $f(\rho, \epsilon)$, which has numerically been determined by us, is a very intriguing result. An interesting question is whether $f(\rho, \epsilon)$ can be determined by other means. Direct experimental observation of $f(\rho, \epsilon)$ in real VRH systems looks impossible. In principle, $f(\rho, \epsilon)$ could be determined by measuring currents in an actually constructed random-resistor network representing a VRH system. The establishment of the scaling found by us and the determination of $f(\rho, \epsilon)$ by theoretical, other than purely numerical, means is a challenge that could further increase our insight into the VRH phenomenon. A fundamental question that remains open is how our results are connected to the percolation approach. The observed scaling, which underlies the temperature dependency of the conductivity in Eq. (1) seems to hold at temperatures where the standard percolation approach is not yet applicable.

\section{ACKNOWLEDGMENTS}

We would like to thank R. Coehoorn for discussions and useful remarks. This work forms part of the research program of the Dutch Polymer Institute (Project No. 274). The computations were made possible by support from NCF (Dutch National Computer Facilities).
${ }^{1}$ For a review see, e.g., T. G. Castner, in Hopping Transport in Solids, edited by M. Pollak and B. I. Shklovskii (North-Holland, Amsterdam, 1991), p. 1.

${ }^{2}$ T. Vuletić, B. Korin-Hamzić, S. Tomić, B. Gorshunov, P. Haas, M. Dressel, J. Akimitsu, T. Sasaki, and T. Nagata, Phys. Rev. B 67, 184521 (2003).

${ }^{3}$ H. C. F. Martens, I. N. Hulea, I. Romijn, H. B. Brom, W. F. Pasveer, and M. A. J. Michels, Phys. Rev. B 67, 121203(R) (2003).

${ }^{4}$ J. A. Reedijk, H. C. F. Martens, H. B. Brom, and M. A. J. Michels, Phys. Rev. Lett. 83, 3904 (1999).

${ }^{5}$ Z. G. Yu and X. Song, Phys. Rev. Lett. 86, 6018 (2001).
${ }^{6}$ N. F. Mott, J. Non-Cryst. Solids 1, 1 (1968); Philos. Mag. 19, 835 (1969)

${ }^{7}$ We deviate here from the usual definition $\alpha=1 / a$.

${ }^{8}$ A. Miller and E. Abrahams, Phys. Rev. 120, 745 (1960).

${ }^{9}$ Depending on the specific type of localized wave function, there may be an additional prefactor in Eq. (3) containing and algebraic dependence on $R_{i j}$. This leads to a different preexponential factor in Eq. (1) (Ref. 12). In the present work, however, we concentrate on the exponential dependence in Eq. (1) and therefore we omit this prefactor.

${ }^{10}$ V. Ambegaokar, B. I. Halperin, and J. S. Langer, Phys. Rev. B 4, 2612 (1971). 
${ }^{11}$ M. Pollak, J. Non-Cryst. Solids 11, 1 (1972).

${ }^{12}$ B. I. Shklovskii and A. L. Efros, Electronic Properties of Doped Semiconductors (Springer, Berlin, 1985).

${ }^{13}$ V. K. S. Shante, Phys. Rev. B 16, 2597 (1977).

${ }^{14}$ A. S. Skal and B. I. Shklovskii, Sov. Phys. Semicond. 8, 1029 (1975).

${ }^{15}$ P. G. de Gennes, La Recherche 72, 919 (1976).

${ }^{16}$ H. E. Stanley, J. Phys. A 10, L211 (1977).

${ }^{17}$ D. N. Tsigankov and A. L. Efros, Phys. Rev. Lett. 88, 176602 (2002).

${ }^{18}$ V. I. Arkhipov, E. V. Emelianova, and G. J. Adriaenssens, Phys. Rev. B 65, 165110 (2002).

${ }^{19}$ T. G. Castner, Phys. Rev. B 61, 16596 (2000).

${ }^{20}$ N. V. Lien and R. Rosenbaum, Phys. Rev. B 56, 14960 (1997).

${ }^{21}$ M. C. J. M. Vissenberg and M. J. M. de Jong, Phys. Rev. Lett. 77, (1996) 4820.

${ }^{22}$ B. Movaghar and W. Schirmacher, J. Phys. C 14, 859 (1981).
${ }^{23}$ O. Bleibaum, H. Böttger, and V. V. Bryksin, Phys. Rev. B 54, 5444 (1996).

${ }^{24}$ D. Bourbie, Philos. Mag. B 73, 201 (1996).

${ }^{25}$ H. Böttger and V. V. Bryksin, Hopping Conduction in Solids (Weinheim, VCH Verlagsgesellschaft, Berlin, 1985).

${ }^{26}$ M. Pollak and B. I. Shklovskii, Hopping Transport in Solids (North-Holland, Amsterdam, 1991).

${ }^{27}$ V. Ambegaokar, S. Cochran, and J. Kurkijärvi, Phys. Rev. B 8, 3682 (1973).

${ }^{28}$ G. E. Pike and C. H. Seager, Phys. Rev. B 10, 1421 (1974).

${ }^{29}$ J. A. McInnes and P. N. Butcher, Philos. Mag. B 39, 1 (1979).

${ }^{30}$ A. S. Skal and B. I. Shklovskii, Sov. Phys. Solid State 16, 1190 (1971); the numbers $c_{2}$ and $c_{3}$ from this paper should be corrected for our different definition of $\alpha$.

${ }^{31}$ D. Monroe, Phys. Rev. Lett. 54, 146 (1985).

${ }^{32}$ S. D. Baranovskii, T. Faber, F. Hensel, and P. Thomas, J. Phys.: Condens. Matter 9, 2699 (1997). 